\title{
Transient instability upon temperature quench in weakly ordered block copolymers
}

\author{
Shuyan Qi \\ Department of Chemical Engineering, University of California, Berkeley, California 94610 \\ Zhen-Gang Wang \\ Division of Chemistry and Chemical Engineering, California Institute of Technology, Pasadena, \\ California 91125
}

(Received 22 July 1999; accepted 17 September 1999)

\begin{abstract}
We report a novel transient instability upon temperature quench in weakly ordered block copolymer microphases possessing a soft direction or directions, such as the lamellar and hexagonal cylinder (HEX) phases. We show that reequilibration of the order parameter is accompanied by transient long wavelength undulation of the layers or cylinders-with an initial wavelength that depends on the depth of the temperature quench-that eventually disappears as the structure reaches its equilibrium at the new temperature. Such undulation leads to a transient transverse broadening of the scattering peaks near the Bragg positions. We argue that this instability might be responsible for the experimentally observed unusual ordering dynamics of the HEX phase of a diblock copolymer after quenching from the disordered state. (C) 1999 American Institute of Physics. [S0021-9606(99)50147-5]
\end{abstract}

\section{INTRODUCTION}

Block copolymers exhibit a variety of ordered microphases with periodicities typically on the order of $10 \mathrm{~s}$ of nanometers. ${ }^{1}$ The relative ease in the control of the molecular characteristics in these systems, such as the molecular weights, composition, and architecture, the softness of interactions in these systems, as well as the long relaxation times generally associated with polymers, make block copolymers ideal model systems for understanding the physics of selfassembly in complex fluids. Recently, ordered block copolymer arrays have been exploited for making novel nanostructured materials. ${ }^{2,3}$

Through extensive experimental and theoretical research in the last two decades, the physics for the phase behavior of block copolymers under equilibrium conditions is now well understood. ${ }^{1}$ In recent years, several groups have also investigated issues concerning the kinetic pathways in various order-order and order-disorder transitions of these selfassembly fluids. ${ }^{4-8}$ Laradji et al., ${ }^{9}$ and independently the present authors, ${ }^{10}$ developed theories of anisotropic fluctuations in ordered block copolymer phases. The instability associated with these anisotropic fluctuations can be used to understand the initial structural evolution in transitions between different ordered morphologies and from an ordered phase to the disordered phase.

In this article, we report a new type of instability that arises when a weakly microphase ordered block copolymer phase with at least one soft direction, such as the lamellar (LAM) or hexagonal cylinder (HEX) phases, is suddenly cooled to a low temperature within the same morphology, or by extrapolation, is obtained by quenching from the disordered phase. We predict a transient enhancement of long wavelength fluctuations in the soft directions. For the sake of simplicity in the algebra, we focus on the LAM phase of diblock copolymers in the main body of the article. However, the qualitative conclusion is equally valid for the HEX phase, and in the Appendix, we briefly demonstrate the instability in the HEX phase using the phase dynamics formulation.

In the following, we first present a linear stability analysis using a simplified Leibler free energy functional ${ }^{11}$ with conserved order-parameter dynamics to demonstrate the nature of the instability. We then calculate the nonequilibrium structure factor of a quenched LAM phase of diblock copolymers to show the transient broadening of the scattering peaks as a result of the aforementioned instability. The latter provides the basis for possible experimental verification of the predicted instability.

\section{THEORY}

We start with a simplified free energy functional for a diblock copolymer melt of the form: ${ }^{11}$

$$
\begin{aligned}
F[\psi(\vec{k})]= & \frac{1}{2} \int d \vec{k} \Gamma_{2}(k) \psi(\vec{k}) \psi(-\vec{k}) \\
& +\frac{1}{3 !} \gamma_{3} \int d\left(\vec{k}_{1} \vec{k}_{2}\right) \psi\left(\vec{k}_{1}\right) \psi\left(\vec{k}_{2}\right) \psi\left(-\vec{k}_{1}-\vec{k}_{2}\right) \\
& +\frac{1}{4 !} \gamma_{4} \int d\left(\vec{k}_{1} \vec{k}_{2} \vec{k}_{3}\right) \psi\left(\vec{k}_{1}\right) \psi\left(\vec{k}_{2}\right) \psi\left(\vec{k}_{3}\right) \\
& \times \psi\left(-\vec{k}_{1}-\vec{k}_{2}-\vec{k}_{3}\right),
\end{aligned}
$$

where $\psi(\vec{k})$ is the Fourier transform of the order parameter $\psi(\vec{r})$ defined as $\phi_{A}(\vec{r})-f_{A}$ with $\phi_{A}(\vec{r})$ and $f_{A}$, respectively, the local and global volume fraction of type $A$ monomers in the diblock copolymer melts. The two point vertex function has the form $\Gamma_{2}(k)=S_{0}^{-1}(k)-2 N \chi$ where $N$ is the polymer- 
ization index, $\chi$ is the Flory-Huggins parameter, and $S_{0}(k)$ is the structure factor for a noninteracting diblock copolymer and is peaked at a wave-number $k_{0}$ which roughly corresponds to the radius of gyration of the diblock copolymer chain but is generally dependent on $f_{A}$. Following Fredrickson and Helfand, ${ }^{12}$ the third- and fourth-order vertex functions have been approximated as $k$-independent constants which are denoted simply by $\gamma_{3}$ and $\gamma_{4}$, respectively. Note that $\gamma_{3}$ and $\gamma_{4}$ are functions of $f_{A} ; \gamma_{3}=0$ when $f_{A}=1 / 2$.

We assume that the time evolution of the order parameter is described by the time-dependent Ginzburg-Landau equation for a conserved order-parameter (model $\mathrm{B}$ in the classification by Hohenberg and Halperin ${ }^{13}$ ) with a constant mobility coefficient $M$. In Fourier space, the equation is

$$
\frac{\partial \psi(\vec{k})}{\partial t}=-k^{2} M\left[\frac{\delta F}{\delta \psi(-\vec{k})}\right]+\eta(\vec{k}, t),
$$

where $\eta(\vec{k}, t)$ is a random force with zero mean and a second moment obeying the fluctuation-dissipation relation

$$
\left\langle\eta(\vec{k}, t) \eta\left(\overrightarrow{k^{\prime}}, t^{\prime}\right)\right\rangle=2(2 \pi)^{3} k_{B} T M k^{2} \delta\left(\vec{k}+\overrightarrow{k^{\prime}}\right) \delta\left(t-t^{\prime}\right) .
$$

In the case of temperature quench, $T$ should be taken as the final temperature. ${ }^{14}$ Equations (1) and (2) have been the basis for several theoretical studies of dynamic issues in block copolymers. ${ }^{15-20}$

In the ordered state, it is convenient to separate the order parameter into an average part and a fluctuating part,

$$
\psi(\vec{k})=\langle\psi(\vec{k})\rangle+\Delta \psi(\vec{k}) .
$$

The average order-parameter can be represented as

$$
\langle\psi(\vec{k})\rangle=\sum_{\vec{G}} A_{\vec{G}} \delta(\vec{k}-\vec{G}),
$$

corresponding to a superposition of plane waves in real space $\langle\psi(\vec{r})\rangle=\Sigma_{\vec{G}} A_{\vec{G}} \exp (i \vec{G} \cdot \vec{r})$, where $\vec{G}$ is the set of reciprocal lattice wave-vectors of the ordered phase. For the LAM phase of diblock copolymers, substituting Eq. (4) into the dynamic equation Eq. (2), we obtain, to linear order in $\Delta \psi(\vec{k})$

$$
\begin{aligned}
\frac{\partial A_{\vec{G}}}{\partial t}= & -M k_{0}^{2}\left[\Gamma_{2}(\vec{G})+\frac{\gamma_{3}}{2} \sum_{\vec{G}^{\prime}} A_{\vec{G}^{\prime}} A_{\vec{G}-\vec{G}^{\prime}}\right. \\
& \left.+\vec{G}^{\prime} A_{\vec{G}^{\prime \prime}} A_{\vec{G}-\vec{G}^{\prime}-\vec{G}^{\prime \prime}}\right],
\end{aligned}
$$

and

$$
\begin{aligned}
\frac{\partial \Delta \psi(\vec{k})}{\partial t}= & -M k^{2}\left[\Gamma_{2}(k) \Delta \psi(\vec{k})+\gamma_{3} \sum_{\vec{G}} A_{\vec{G}} \Delta \psi(\vec{k}-\vec{G})\right. \\
& \left.+\frac{\gamma_{4}}{2} \sum_{\vec{G}} \sum_{\vec{G}^{\prime}} A_{\vec{G}^{\prime}} A_{\vec{G}^{\prime}} \Delta \psi\left(\vec{k}-\vec{G}-\vec{G}^{\prime}\right)\right]+\eta(\vec{k}, t) .
\end{aligned}
$$

It is obvious that $1 / k_{0}$ serves as a natural length scale in our problem, while $1 /\left(M k_{0}^{2}\right)$ yields a natural time scale.
Thus, it is convenient to define dimensionless wave-vectors $\vec{k} / k_{0}$ and dimensionless time $t M k_{0}^{2}$. However, to avoid excessiveness of notations, we will denote these simply as $\vec{k}$ and $t$, with the understanding that in the subsequent analyses, the units of time and wave-numbers are, respectively, $1 /\left(M k_{0}^{2}\right)$ and $k_{0}$.

In the weak segregation limit, the most important terms in the order parameter Eq. (5) are those with wave-vectors $|\vec{G}|=1$ (in units of $k_{0}$ ). For the lamellar phase there are only two such wave-vectors $\vec{G}=\hat{z}$ and $\vec{G}=-\hat{z}$, where we have taken the lamellar normal in the $z$-direction. We denote these two wave-vectors as $\vec{G}_{0}$ and $-\vec{G}_{0}$, respectively. With a proper choice of the origin of $z$, we can choose $A_{\vec{G}_{0}}=A_{-\vec{G}_{0}}$ $=A$ to be real. Thus, Eqs. (6) and (7) become,

$$
\frac{\partial A}{\partial t}=-\left(\tau+\frac{1}{2} \gamma_{4} A^{2}\right) A,
$$

and

$$
\begin{aligned}
\frac{\partial \Delta \psi(\vec{k})}{\partial t}= & -k^{2}\left\{\Gamma_{2}(k) \Delta \psi(\vec{k})\right. \\
& +\gamma_{3} A\left[\Delta \psi\left(\vec{k}-\vec{G}_{0}\right)+\Delta \psi\left(\vec{k}+\vec{G}_{0}\right)\right] \\
& +\frac{\gamma_{4}}{2} A^{2}[2 \Delta \psi(\vec{k}) \\
& \left.\left.+\Delta \psi\left(\vec{k}-2 \vec{G}_{0}\right)+\Delta \psi\left(\vec{k}+2 \vec{G}_{0}\right)\right]\right\}+\eta(\vec{k}, t),
\end{aligned}
$$

where we have defined $\tau=\Gamma_{2}\left(k_{0}\right)=2\left(N \chi^{*}-N \chi\right)$ with $\chi^{*}$ the value of $\chi$ at the spinodal of the disordered phase. For an instantaneous temperature quench, the final $\tau$ value should be used.

Equation (9) represents a set of coupled equations that describe the time evolution of the anisotropic fluctuations in the LAM phase. These equations can be diagonalized to obtain the largest fluctuation modes ${ }^{10}$ at a given temperature. Alternatively, they can be analyzed using the standard perturbation arguments. ${ }^{21}$ For asymmetric diblocks $\left(\gamma_{3} \neq 0\right)$, Eq. (9) predicts that the least stable modes near the spinodal of the LAM with respect to the HEX are on two rings in the reciprocal space located at $|\vec{k}|=1$ and $k_{z}= \pm 1 / 2 .{ }^{9,10}$ In this paper, however, we are interested in long wavelength instabilities in the lamellar structure upon a temperature quench. Thus, we only examine fluctuations with $\vec{k}$ near $\vec{G}_{0}$. Furthermore, although the analysis will not be presented here, it can be shown that the most unstable long wavelength fluctuations are those with wave-vectors perpendicular to the direction of $\vec{G}_{0}$. Therefore, we focus on instabilities with wavevectors $\vec{k}= \pm \vec{G}_{0}+\vec{q}$ where $\vec{q}$ is perpendicular to $\vec{G}$. The most significant fluctuations we need to keep are of the form $\Delta \psi\left(\vec{G}_{0}+\vec{q}\right)$ and $\Delta \psi\left(\vec{q}-\vec{G}_{0}\right)$. The time evolution of each is obtained by setting $\vec{k}=\vec{G}_{0}+\vec{q}$ and $\vec{k}=-\vec{G}_{0}+\vec{q}$, respectively, in Eq. (9), which yields a set of coupled equations: 


$$
\begin{aligned}
\frac{\partial \Delta \psi\left(\vec{G}_{0}+\vec{q}\right)}{\partial t}= & -k^{2}\left\{\left[\Gamma_{2}(k)+\gamma_{4} A^{2}\right] \Delta \psi\left(\vec{G}_{0}+\vec{q}\right)\right. \\
& \left.+\frac{\gamma_{4}}{2} A^{2} \Delta \psi\left(\vec{G}_{0}-\vec{q}\right)\right\}+\eta\left(\vec{G}_{0}+\vec{q}, t\right), \quad(10) \\
\frac{\partial \Delta \psi\left(-\vec{G}_{0}+\vec{q}\right)}{\partial t}= & -k^{2}\left\{\left[\Gamma_{2}(k)+\gamma_{4} A^{2}\right] \Delta \psi\left(-\vec{G}_{0}+\vec{q}\right)\right. \\
& \left.+\frac{\gamma_{4}}{2} A^{2} \Delta \psi\left(\vec{G}_{0}+\vec{q}\right)\right\}+\eta\left(-\vec{G}_{0}+\vec{q}, t\right),
\end{aligned}
$$

where we have made use of the fact that $k^{2}=\left(\vec{G}_{0}+\vec{q}\right)^{2}$ $=\left(\vec{G}_{0}-\vec{q}\right)^{2}$. (Note that terms involving $\gamma_{3}$ do not appear in Eqs. (10a) and (10b): they involve $\Delta \psi(\vec{q}), \Delta \psi\left(-2 \vec{G}_{0}\right.$ $+\vec{q})$, and $\Delta \psi\left(2 \vec{G}_{0}+\vec{q}\right)$ which are negligible for small $|\vec{q}|$, as compared to the dominant fluctuation modes at $\vec{k}= \pm \vec{G}_{0}$ $+\vec{q}$.) Diagonalizing these equations, we obtain two eigenmodes with their associated growth rates (eigenvalues). The first eigenmode corresponds to $\Delta \psi\left(\vec{G}_{0}+\vec{q}\right): \Delta \psi\left(-\vec{G}_{0}+\vec{q}\right)$ $=1:-1$ and the second eigenmode corresponds to $\Delta \psi\left(\vec{G}_{0}\right.$ $+\vec{q}): \Delta \psi\left(-\vec{G}_{0}+\vec{q}\right)=1: 1$, with the respective eigenvalues

$$
\begin{aligned}
& \sigma_{1}(k)=-k^{2}\left[\Gamma_{2}(k)+\frac{\gamma_{4}}{2} A^{2}\right], \\
& \sigma_{2}(k)=-k^{2}\left[\Gamma_{2}(k)+\frac{3 \gamma_{4}}{2} A^{2}\right] .
\end{aligned}
$$

Before we proceed to analyze the stability of these modes, it is instructive to understand their physical content. In position space, the first eigenmode corresponds to a fluctuation of the form:

$$
\begin{aligned}
\Delta \psi_{1}(\vec{r}) & =c_{1}\left[\cos \left(z+\vec{q} \cdot \vec{r}_{\|}\right)-\cos \left(z-\vec{q} \cdot \vec{r}_{\|}\right)\right] \\
& =-2 c_{1} \sin (z) \sin \left(\vec{q} \cdot \vec{r}_{\|}\right),
\end{aligned}
$$

where $\vec{r}_{\|}$is the position vector in the plane of the layers. When added to the mean field order-parameter $\langle\psi(\vec{r})\rangle$ $=2 A \cos (z)$, this yields an order parameter profile of the form

$$
\begin{aligned}
\psi_{1}(\vec{r}) & =2 A \cos (z)-2 c_{1} \sin (z) \sin \left(\vec{q} \cdot \vec{r}_{\|}\right) \\
& \approx 2 A \cos \left(z+u\left(\vec{r}_{\|}\right)\right)
\end{aligned}
$$

where $u\left(\vec{r}_{\|}\right) \approx\left(c_{1} / A\right) \sin (\vec{q} \cdot \vec{r} \mid)$. Obviously, the above equation describes a lamellar structure with undulation of the layers in the directions transverse to the lamellar normal.

A similar analysis shows that the second eigenmode leads to an order parameter profile of the form

$$
\psi_{2}(\vec{r})=2\left[A+c_{2} \cos \left(\vec{q} \cdot \vec{r}_{\|}\right)\right] \cos (z),
$$

which describes a lamellar structure with the amplitude modulated periodically in the directions parallel to the layers. These two modes are illustrated schematically in Fig. 1.
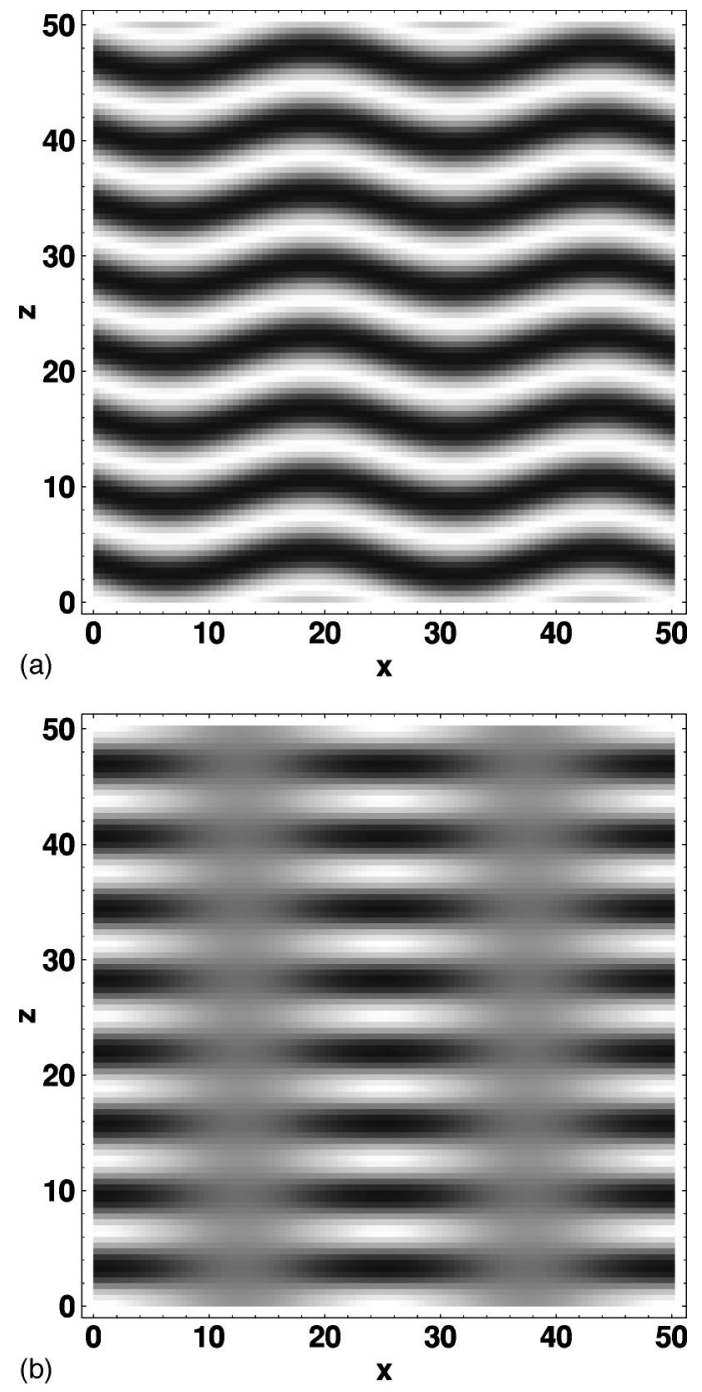

FIG. 1. A two-dimensional illustration of the (a) undulation mode and (b) the amplitude-modulation mode, corresponding to Eqs. (13) and (14), respectively. The parameters are set to be $A=0.5, c_{1}=0.25, c_{2}=0.376$, and $|\vec{q}|=0.25$.

We now investigate the stability of these modes. Consider first the undulation mode. To facilitate the analysis and make the results more transparent, we use an approximate form of $\Gamma_{2}$ in Eqs. (11a), (11b) by taking $\Gamma_{2}(k)=\tau+b(k$ $-1 / k)^{2}$ where $b$ is a $f_{A}$-dependent coefficient of order one that can be calculated from the exact $\Gamma_{2}(k)$ near $k_{0}$ (taken to be 1 in our units). This simple form captures the correct behavior of $\Gamma_{2}$ near $k_{0}$ as well as its asymptotic behavior in both the small and large $k$ limits. ${ }^{11,22,23}$ The exact $\Gamma_{2}(k)$ can be used without any difficulty, which only leads to insignificant quantitative differences from the results obtained using the approximate form. Use of this approximate form of $\Gamma_{2}$ yields a simple expression for the growth rate $\sigma_{1}$ :

$$
\sigma_{1}=-\tau-\frac{1}{2} \gamma_{4} A^{2}-\left(\tau+\frac{1}{2} \gamma_{4} A^{2}\right) q^{2}-b q^{4}
$$

For a lamellar phase at equilibrium, from Eq. (8), the amplitude of the lamellar wave satisfies $\tau+\frac{1}{2} \gamma_{4} A^{2}=0$. So the growth rate is simply $\sigma_{1}=-b q^{4}$ and is negative. Thus, 
any undulation in the layers will be damped. Physically this damping is due to the bending free energy penalty for the lamellar phase at equilibrium.

On the other hand, when temperature is suddenly decreased from an initial value $\tau_{i}$ to a lower (more negative) $\tau_{f}$ corresponding to a final equilibrium value $A_{f}=\sqrt{-2 \tau_{f} / \gamma_{4}}$, the above growth rate becomes

$$
\sigma_{1}=\frac{1}{2} \gamma_{4}\left[A_{f}^{2}-A(t)^{2}\right]+\frac{1}{2} \gamma_{4}\left[A_{f}^{2}-A(t)^{2}\right] q^{2}-b q^{4}
$$

where $A(t)$ evolves according to Eq. (8) with an initial amplitude $A_{i}=\sqrt{-2 \tau_{i} / \gamma_{4}}$. Obviously, $A_{f}^{2}-A^{2}>0$ for all $t>0$ and vanishes only as $t \rightarrow \infty$. In fact, the amplitude Eq. (8) can be solved analytically to yield

$$
A(t)^{2}=\frac{A_{i}^{2} \exp \left(\gamma_{4} A_{f}^{2} t\right)}{1+\left(A_{i} / A_{f}\right)^{2} \exp \left(\gamma_{4} A_{f}^{2} t\right)}
$$

Thus we have an analytical expression for the timedependence of the growth rate.

The $q$-independent term in Eq. (16) is due to the growth of the amplitude of the lamellar wave, whereas the $q$-dependent terms give rise to spatial structures in the transverse directions. At time $t$, the growth rate is positive for wave-numbers within $0<q<q_{m}$ with a maximum value at $q=q_{o}$, where

$$
\begin{aligned}
q_{m}= & \frac{1}{2 \sqrt{b}}\left[\gamma_{4}\left(A_{f}^{2}-A^{2}\right)\right. \\
& \left.+\sqrt{\gamma_{4}^{2}\left(A_{f}^{2}-A^{2}\right)^{2}+8 b \gamma_{4}\left(A_{f}^{2}-A^{2}\right)}\right]^{1 / 2},
\end{aligned}
$$

and

$$
q_{o}=\left[\gamma_{4}\left(A_{f}^{2}-A^{2}\right) /(2 b)\right]^{1 / 2} .
$$

Thus, any undulation with wave-numbers in the range $0<q$ $<q_{m}$ is unstable, that with $q_{o}$ corresponding to an undulation mode that grows the fastest. Note that as $A(t)$ evolves towards its new equilibrium value, $q_{m}$ and $q_{o}$ shift to smaller values and eventually vanish.

A similar analysis of $\sigma_{2}$ shows that the mode corresponding to Eq. (14) becomes unstable when $A_{f}^{2}>3 A^{2}$. Thus this instability requires a deeper temperature quench and also disappears at an earlier time.

In the Appendix, we provide a different view of the undulation instability by using Eq. (13) as an ansatz and derive a dynamic equation for the phase $u\left(\vec{r}_{\|}\right)$.

To more fully characterize the fluctuations, we calculate the nonequilibrium structure factor $S\left(\vec{G}_{0}+\vec{q},-\vec{G}_{0}-\vec{q} ; t\right)$ $=\left\langle\Delta \psi\left(\vec{G}_{0}+\vec{q}, t\right) \Delta \psi\left(\vec{G}_{0}+\vec{q}, t\right)\right\rangle$. From Eqs. (10a), (10b), we can derive a set of two coupled equations involving $S\left(\vec{G}_{0}\right.$ $\left.+\vec{q},-\vec{G}_{0}-\vec{q} ; t\right)$ and the off-diagonal $S\left(\vec{G}_{0}+\vec{q},-\vec{G}_{0}+\vec{q} ; t\right)$ which we will denote as $S(q, t)$ and $H(q, t)$, respectively. These equations are first diagonalized and then solved using standard methods for linear, first-order differential equations. We skip the algebra here and merely quote the solution for $S(q, t)$ which is the experimentally measurable quantity

$$
\begin{aligned}
S(q, t)= & \exp \left[\Lambda_{1}(q, t)\right]\left\{\frac{1}{2}\left[S_{0}(q)-H_{0}(q)\right]\right. \\
& \left.+\frac{1+q^{2}}{(2 \pi)^{3}} \int_{0}^{t} d t^{\prime} \exp \left[-\Lambda_{1}\left(q, t^{\prime}\right)\right]\right\} \\
& +\exp \left[\Lambda_{2}(q, t)\right]\left\{\frac{1}{2}\left[S_{0}(q)-H_{0}(q)\right]\right. \\
& \left.+\frac{1+q^{2}}{(2 \pi)^{3}} \int_{0}^{t} d t^{\prime} \exp \left[-\Lambda_{2}\left(q, t^{\prime}\right)\right]\right\},
\end{aligned}
$$

where $S_{0}(q)$ and $H_{0}(q)$ are the equilibrium diagonal and off-diagonal structure factors at the initial $\tau$, and the functions $\Lambda_{1}(q, t)$ and $\Lambda_{2}(q, t)$ are defined as

$$
\begin{aligned}
& \Lambda_{1}(q, t)=2 \int_{0}^{t} d t^{\prime} \sigma_{1}\left(q, t^{\prime}\right), \\
& \Lambda_{2}(q, t)=2 \int_{0}^{t} d t^{\prime} \sigma_{2}\left(q, t^{\prime}\right) .
\end{aligned}
$$

The integrals can be performed by using the expressions for $\sigma_{1}$ and $\sigma_{2}$ in combination with the solution for the amplitude Eq. (8); the results are

$\Lambda_{1}(q, t)=-2 b q^{4} t+\left(1+q^{2}\right) \ln \left[\frac{A(t)}{A_{i}}\right]^{2}$,
$\Lambda_{2}(q, t)=-2 b q^{4} t+4 \tau_{f}\left(1+q^{2}\right)+3\left(1+q^{2}\right) \ln \left[\frac{A(t)}{A_{i}}\right]^{2}$,

where the ratio involving $A(t)$ is given by Eq. (17).

The calculated structure factor at different times is shown in Fig. 2 for a temperature quench from $\tau_{i}=-0.2$ to $\tau_{f}=-1.5$ corresponding to a change in $N \chi$ from 10.6 to 12 . Figure 2(a) shows the structure factor $S(q, t)$ while Fig. 2(b) shows the ratio $S(q, t) / S(q, 0)$. The enhancement of scattering at intermediate times is evident in both plots. In Fig. 2(b), a shallow maximum can be observed in the enhancement, which corresponds to the fastest growing mode. As time evolves, the large $q$-part of the scattering curves collapses to the equilibrium scattering function at the final $\tau_{f}$. This reflects the suppression of fluctuations at large $q$ 's. This "cutting-off" behavior can be understood by examining the function $\Lambda_{1}(q, t)$. Roughly speaking, the sign of $\Lambda_{1}(q, t)$ can serve as indicator of whether a fluctuation with wave-number $q$ is enhanced or suppressed. Since $A(t)$ approaches its final equilibrium value exponentially [see Eq. (17) and Fig. 2(c)], for large $t$, we may set $A(t)=A_{f}$ in Eqs. (22a). Setting $\Lambda_{1}$ $=0$, we obtain

$$
q_{c} \approx\left[b^{-1} \ln \left(A_{f} / A_{i}\right)\right]^{1 / 4} t^{-1 / 4},
$$

while the wave-number corresponding to the largest enhancement shifts to zero according to

$$
q_{f}=\left[\frac{1}{2 b} \ln \left(A_{f} / A_{i}\right)\right]^{1 / 2} t^{-1 / 2} .
$$




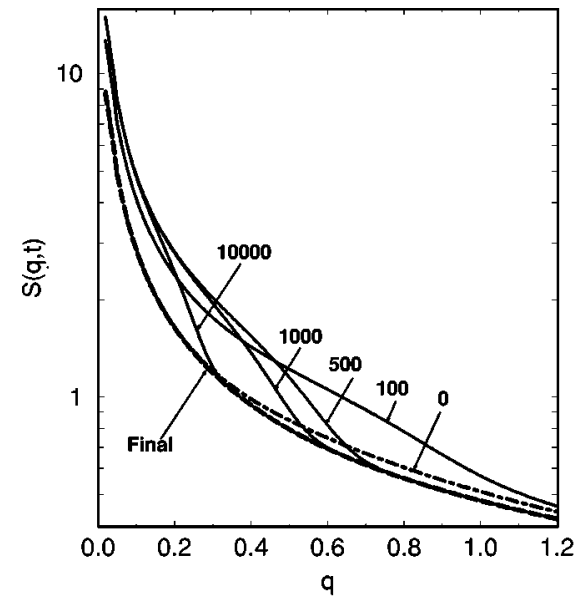

(a)

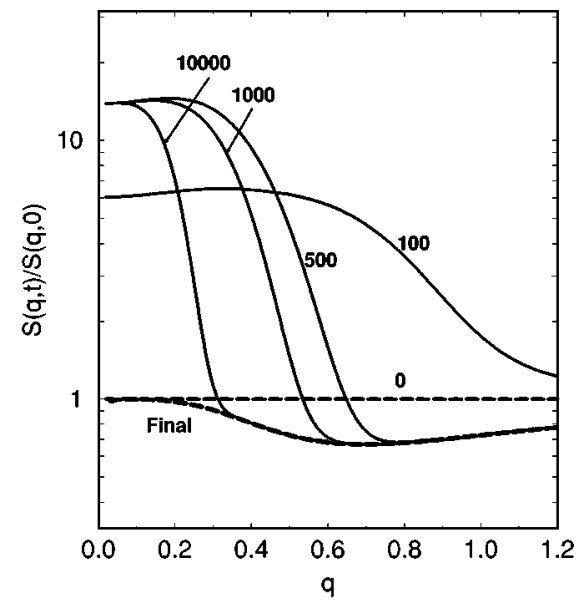

(b)

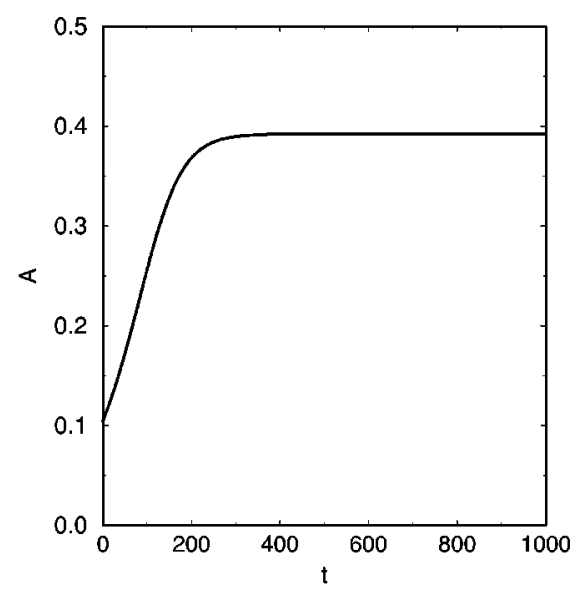

(c)

FIG. 2. Evolution of (a) the structure factor $S(q, t)$ and (b) the ratio $S(q, t) / S(q, 0)$ for a symmetric diblock copolymer after a temperature quench from $N \chi_{i}=10.6$ to $N \chi_{f}=12$ within the lamellar state. (c) Evolution of the amplitude $A(t)$ of the lamellar wave for the same temperature quench. For the structure factor, the exact form of $\Gamma_{2}(k)$ is used.

Thus, the enhanced scattering decays algebraically even though the order parameter approaches equilibrium exponentially. This is clearly seen in Fig. 2: While the amplitude all but reaches its final equilibrium value at $t=300$, considerable enhancement of the scattering at small $q$ remains for $t$ $=10000$.

These predictions can be directly tested in experiments using well-aligned lamellar samples.

\section{DISCUSSION AND CONCLUSION}

It should be clear from our analysis in Sec. II that the origin of the undulation instability is not fundamentally different from the better-known instability in the spinodal decomposition of binary fluid mixtures. ${ }^{24}$ However, the special symmetry in the lamellar phase makes the directions transverse to the lamellar normal different from the normal direction. Specifically, for long wavelength structural fluctuations at equilibrium, the deformation (measured by the local displacement of the layers from their equilibrium positions) can be separated into a longitudinal (compression) mode and a transverse (bending or undulation) mode. ${ }^{25-28}$ While the former is penalized by a free energy cost proportional to the square of the first derivative of the displacement in the layer normal direction, the bending term is penalized only by the square of the second derivative in the transverse directions, therefore making the bending mode and the transverse directions especially "soft" and thus most susceptible to long wavelength perturbations. A temperature quench in effect produces a negative square gradient term for the displacement in the free energy. Whereas a longitudinal instability is prevented by the existence of the positive square gradient term in the longitudinal direction at equilibrium, no such term exists in the transverse direction and as a result an undulation instability develops for sufficiently long wavelength perturbations. A similar argument can be used to understand the undulation instability of the cylinders in the HEX phase.

Although our analysis assumes an initial state that is already in the lamellar phase, mathematically, our results should be equally valid when the initial state is the disordered state corresponding to a special $A(0)=0$. (Technically a small perturbation is needed to set the deterministic equation Eq. (8) in motion.) Figure 3 shows the enhancement of the scattering for a temperature quench corresponding to a change of $N \chi$ from 0 to 12 . In this case the effects are seen to be much more pronounced than for a quench within the lamellar phase as shown in Fig. 2. Again, the enhancement of the scattering at small wave-numbers persists long after the amplitude has reached its equilibrium value.

The Fredrickson-Helfand theory ${ }^{12}$ predicts that the transition from the disordered state to an ordered state always involves a free energy barrier for finite polymerization index $N$ and hence the transition kinetics from the disordered to the lamellar phase will always be via nucleation and growth. ${ }^{29}$ This conclusion, however, is based on a specific method for treating the nonlinear fluctuations in the disordered states. While a number of experiments support the nucleation and growth mechanism, ${ }^{30-32}$ two recent experiments ${ }^{33,34}$ seem to suggest the possibility of either spinodal-like kinetics, or vanishingly small nucleation barrier, in the disorder-order transition in block copolymers. A clear experimental distinction between the two scenarios may not be possible; what is relevant to our present discussion is that the lamellar or hexagonal order can appear on a large scale and without any significant time delay. Then, the ordering process can be viewed as a combination of the selection of the orientation of the lamellae or cylinders and the growth of the amplitude of the density wave. Our study thus shows that a newly formed LAM or HEX structure cannot be perfectly aligned but will be modulated by the undulation of the layers or cylinders in the soft directions and that these undulations can persist long after the lamellar or HEX order (measured by the amplitude of the density wave) is well established. An upper limit of the initial coherence length $L$ in the transverse direction (along the cylinder direction or normal to the layer direction) can be estimated. From the Appendix and setting $A=0$ in Eq. (A8), we get 


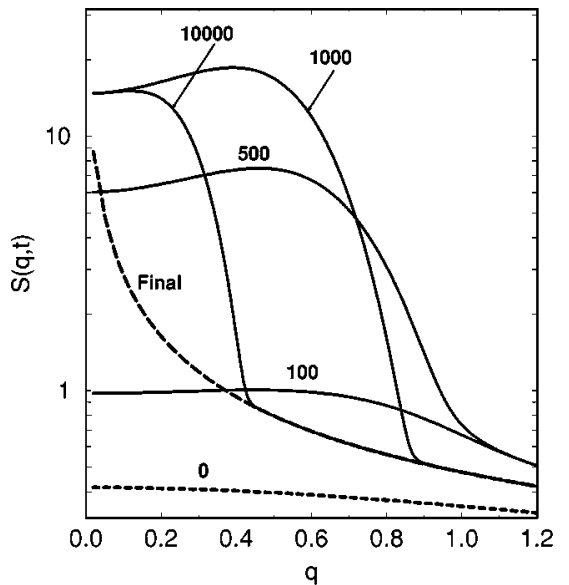

(a)

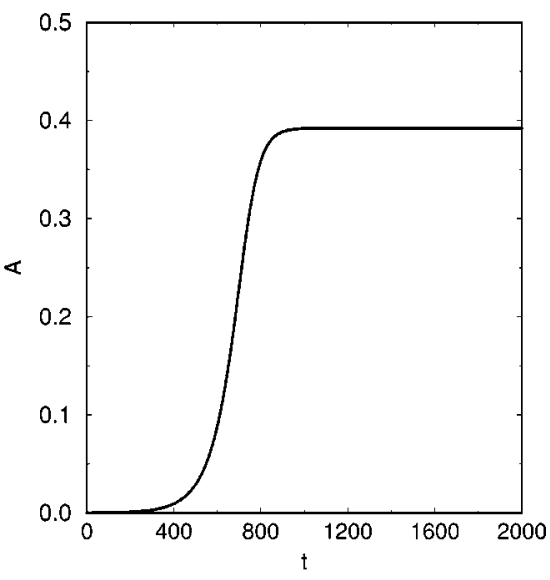

(b)

$$
L=\frac{2 \pi}{k_{0}} \sqrt{\frac{b}{2\left(N \chi_{f}-N \chi^{*}\right)}} .
$$

Our results might offer a possible explanation of the unusual kinetics observed by Balsara et al. ${ }^{34}$ in a temperature quench from the disordered state to the HEX state for polystyrene-b-polyisoprene copolymer melts. These authors report that for large quench depths, the transition kinetics exhibit spinodal-like behavior. Using a combination of small-angle $\mathrm{x}$-ray scattering and time-resolved depolarized light scattering, they demonstrate that hexagonal order occurs before the development of a coherent structure along the cylinder direction (which they call the liquid direction). They envisage the formation of arrays of hexagonally packed cylinders with a limited persistent length at the initial stage of the transition and subsequent straightening of the cylinders at late stages, with the second process occurring at a much slower rate. Such wormlike cylinders have been directly observed in a later experiment by Modi et al. ${ }^{35}$ These findings are consistent with our theoretical results.

\section{ACKNOWLEDGMENTS}

We thank Professors N. P. Balsara, M. Muthukumar, and M. Cross for helpful discussions. We particularly thank Professor N. P. Balsara for explaining his experimental findings in Ref. 34 to us which in large part provide the impetus for our work. Financial support from the National Science Foundation (DMR-9531914), the Camille and Henry Dreyfus Foundation (TC-96-063), the Alfred P. Sloan Foundation (BR-3508), and the Department of Energy (BES, through Professor A. K. Chakraborty at UC Berkeley) is gratefully acknowledged.

\section{APPENDIX}

In order to provide additional insight into the nature of the instability and to provide support for our claim that a similar instability also arises in the HEX phase, we briefly sketch a linear stability analysis for the phase dynamics for both the LAM and HEX structures. For this analysis, it is more convenient to work in real space. Substituting the free energy functional into the time-dependent Ginzburg-Landau equation Eq. (2) and Fourier transforming the equation back to real space, we obtain

$$
\frac{\partial \psi}{\partial t}=\nabla^{2}\left[\tau \psi-b \nabla^{2} \psi-2 b \psi+\frac{1}{2} \gamma_{3} \psi^{2}+\frac{1}{6} \gamma_{4} \psi^{3}\right]-b \psi,
$$

where we have used the simplified form for $\Gamma_{2}: \Gamma_{2}(k)=\tau$ $+b(k-1 / k)^{2}$ and scaled the length and time, respectively, by $1 / k_{0}$ and $1 /\left(M k_{0}^{2}\right)$. For the linear stability analysis, we have dropped the thermal noise term.

We first consider the lamellar phase. We take the lamellar normal to be in the z-direction and denote the position vector in the plane parallel to the layers by $\vec{r}_{\|}$. For small displacement of the layers from their perfect periodic position, we may write the order parameter as

$$
\psi(\vec{r})=A \sum_{G} \exp \left\{i G\left[z+u\left(\vec{r}_{\|}, z\right)\right]\right\}
$$

where $u\left(\vec{r}_{\|}, z\right)$ is the local displacement which amounts to a phase shift and $G= \pm 1$. At the linear order of $u$, we may expand the exponential to write

$$
\psi(\vec{r})=A \sum_{G} \exp (i G z)\left[1+i G u\left(\vec{r}_{\|}, z\right)+O\left(u^{2}\right)\right]
$$

For the lamellar phase in the one wave-number approximation, the quadratic term in the dynamic equation (or the cubic term in the free energy) does not contribute because it cannot produce a sinusoidal wave of the chosen wave-number. For the cubic term in the dynamic equation Eq. (A1), we have

$$
\begin{aligned}
\psi^{3}= & A^{3} \sum_{G_{1}} \sum_{G_{2}} \sum_{G_{3}} \exp \left[i\left(G_{1}+G_{2}+G_{3}\right) z\right] \\
& \times\left[1+i\left(G_{1}+G_{2}+G_{3}\right) u\left(\vec{r}_{\|}, z\right)+O\left(u^{2}\right)\right] .
\end{aligned}
$$

The one wave-number approximation restricts the sum to only those values of $G_{i}$ 's that satisfy $G_{1}+G_{2}+G_{3}= \pm 1$. Since each $G_{i}= \pm 1$, the above sum reduces to

$$
\psi^{3}=3 A^{3} \sum_{G} \exp (i G z)\left[1+i G u\left(\vec{r}_{\|}, z\right)+O\left(u^{2}\right)\right]
$$


Substituting Eqs. (A3), (A5) into Eq. (A1), equating the real and imaginary terms separately and using the orthogonality of the function $\exp (i G z)$, we obtain an equation for the amplitude $A$, which is nothing but Eq. (8), and an equation for the phase:

$$
\begin{aligned}
\frac{\partial u}{\partial t}= & \left(\tau+\frac{1}{2} \gamma_{4} A^{2}\right) \nabla_{\|}^{2} u+\left(\tau+\frac{1}{2} \gamma_{4} A^{2}+4 b\right) \frac{\partial^{2} u}{\partial z^{2}} \\
& -b\left(\nabla_{\|}^{2}+\frac{\partial^{2}}{\partial z^{2}}\right)^{2} u .
\end{aligned}
$$

Fourier transforming this equation, we get

$$
\begin{aligned}
\frac{\partial u\left(\vec{q}, k_{z}\right)}{\partial t}= & -\left[\left(\tau+\frac{1}{2} \gamma_{4} A^{2}\right) q^{2}\right. \\
& \left.+\left(\tau+\frac{1}{2} \gamma_{4} A^{2}+4 b\right) k_{z}^{2}+b k^{4}\right] u\left(\vec{q}, k_{z}\right),
\end{aligned}
$$

where $\vec{q}$ and $k_{z}$ are the wave-vectors conjugate to $\vec{r}_{\|}$and $z$, respectively, and $k^{2}=q^{2}+k_{z}^{2}$.

At equilibrium, $\tau+\frac{1}{2} \gamma_{4} A^{2}=0$. Thus fluctuation in $u$ will be damped at a rate $4 b k_{z}^{2}+b k^{4}$. Clearly, the $k_{z}^{2}$ term corresponds to an inhomogeneous compression/dilatation mode. Since this mode is already penalized by the $k_{z}^{2}$ term, higher order terms in $k_{z}$ may be ignored and $4 b k_{z}^{2}+b k^{4} \approx 4 b k_{z}^{2}$ $+b q^{4}$. We recognize the $q^{4}$ term as corresponding to the undulation mode. (In dimensionless form, the ratio between the compression and bending moduli for the lamellar phase is 4 in the weak segregation limit. ${ }^{27}$ )

After a temperature quench, $\tau+\frac{1}{2} \gamma_{4} A^{2}<0$. Thus, an undulation mode with wave-vectors satisfying $q^{2}<q_{c}^{2}$ will be unstable, where

$$
q_{c}^{2}=-\left(\tau+\frac{1}{2} \gamma_{4} A^{2}\right) / b .
$$

The maximum growth rate occurs for $q^{2}=q_{c}^{2} / 2$.

The compression/dilatation mode, on the other hand, remains stable because in the weak segregation we are considering, $|\tau|<b$ and hence $\tau+\frac{1}{2} \gamma_{4} A^{2}+4 b>0$. Therefore, insofar as the most unstable mode is concerned, we can simply restrict our consideration to the pure undulation mode by setting $k_{z}=0$.

We now consider the HEX phase. A similar consideration to the discussion given above leads to the expectation that the most unstable deformation will again be the undulation of cylinders because the compression and shear moduli are both positive at equilibrium. Assuming an ansatz of the following form:

$$
\begin{aligned}
\psi(\vec{r}) & =A \sum_{\vec{G}} \exp \left\{i \vec{G} \cdot\left[\vec{r}_{\perp}+\vec{u}(x)\right]\right\} \\
& =A \sum_{\vec{G}} \exp \left[i \vec{G} \cdot \vec{r}_{\perp}\right]\left\{1+i \vec{G} \cdot \vec{u}(x)+O\left(u^{2}\right)\right\},
\end{aligned}
$$

where $\vec{G}$ 's are the set of the lowest reciprocal lattice vectors for the HEX structure with $|\vec{G}|=1$. The cylinders are assumed to be oriented along the $x$-direction, and $\vec{r}_{\perp}$ is the position vector in the $y-z$ plane. Since we are only interested in the undulation mode, we have suppressed the $\vec{r}_{\perp}$ dependence in $\vec{u}$.

In the one wave-number approximation, $\psi^{2}$ and $\psi^{3}$, are, respectively,

$$
\psi^{2}=2 A^{2} \sum_{\vec{G}} \exp \left[i \vec{G} \cdot \vec{r}_{\perp}\right]\left\{1+i \vec{G} \cdot \vec{u}(x)+O\left(u^{2}\right)\right\},
$$

and

$\psi^{3}=15 A^{3} \sum_{\vec{G}} \exp \left[i \vec{G} \cdot \vec{r}_{\perp}\right]\left\{1+i \vec{G} \cdot \vec{u}(x)+O\left(u^{2}\right)\right\}$.

Substituting these into Eq. (A1), equating the real and imaginary terms separately, and using the orthogonality of the function $\exp \left(i \vec{G} \cdot \vec{r}_{\perp}\right)$, we obtain

$$
\frac{\partial A}{\partial t}=-\left(\tau+\gamma_{3} A+\frac{5}{2} \gamma_{4} A^{2}\right) A,
$$

and

$$
\frac{\partial \theta}{\partial t}=\left(\tau+\gamma_{3} A+\frac{5}{2} \gamma_{4} A^{2}\right) \frac{\partial^{2} \theta}{\partial x^{2}}-b \frac{\partial^{4} \theta}{\partial x^{4}},
$$

where $\theta=\vec{G} \cdot \vec{u}(x)$. An equation of this form exists for each of the $\vec{G}$ 's. However, since only two of the six wave-vectors are independent, there are only two independent equations which are identical in form. Fourier transforming the above equation, we get

$$
\frac{\partial \theta(q)}{\partial t}=\left(\tau+\gamma_{3} A+\frac{5}{2} \gamma_{4} A^{2}\right) q^{2} \theta-b q^{4} \theta .
$$

The analysis of this equation is the same as for the LAM case. After a temperature quench, modes with $q$ in the range of $0<q^{2}<q_{c}^{2}$ are unstable with $q_{c}^{2}$ now given by

$$
q_{c}^{2}=-\left(\tau+\gamma_{3} A+\frac{5}{2} \gamma_{4} A^{2}\right) / b .
$$

${ }^{1}$ F. S. Bates and G. H. Fredrickson, Phys. Today 52, 32 (1999).

${ }^{2}$ S. Förster and M. Antonietti, Adv. Mater. 10, 195 (1998).

${ }^{3}$ J. F. Ding and G. J. Liu, Chem. Mater. 10, 537 (1998)

${ }^{4}$ S. Sakurai, T. Momii, K. Taie, M. Shibayama, S. Nomura, and T. Hashimoto, Macromolecules 26, 485 (1993); S. Sakurai, H. Kawada and T. Hashimoto, ibid. 26, 5796 (1993); N. Sakamoto, T. Hashimoto, C. D. Han, D. Kim, and N. Y. Vaidya, ibid. 30, 1621 (1997).

${ }^{5}$ D. A. Hajduk, S. M. Gruner, P. Rangarajan, R. A. Register, L. J. Fetters, C. Honeker, R. J. Albalak, and E. L. Thomas, Macromolecules 27, 490 (1994).

${ }^{6}$ M. F. Schulz, F. S. Bates, K. Almdal, and K. Mortensen, Phys. Rev. Lett. 73, 86 (1994); K. A. Koppi, M. Tirrell, F. S. Bates, K. Almdal, and K. Mortensen, J. Rheol. 38, 999 (1994); K. Almdal, K. Mortensen, K. A. Koppi, M. Tirrell, and F. S. Bates, J. Phys. II 6, 617 (1996).

${ }^{7}$ C. Y. Ryu, M. S. Lee, D. A. Hajduk, and T. P. Lodge, J. Polym. Sci., Part B: Polym. Phys. 35, 2811 (1997); C. Y. Ryu, M. E. Vilgild, and T. P. Lodge, Phys. Rev. Lett. 81, 5354 (1998).

${ }^{8}$ J. K. Kim, H. H. Lee, Q.-J. Gu, T. Chang, and Y. H. Jeong, Macromolecules 31, 4045 (1998); H. H. Lee and J. K. Kim (unpublished).

${ }^{9}$ C. Yeung, A. C. Shi, J. Noolandi, and R. C. Desai, Macromol. Theory Simul. 5, 291 (1996); M. Laradji, A. C. Shi, R. C. Desai, and J. Noolandi, Phys. Rev. Lett. 78, 2577 (1997); M. Laradji, A. C. Shi, J. Noolandi and R. C. Desai, Macromolecules 30, 3242 (1997).

${ }^{10}$ S. Y. Qi and Z.-G. Wang, Macromolecules 30, 4491 (1997); S. Y. Qi and Z.-G. Wang, Polymer 39, 4639 (1998).

${ }^{11}$ L. Leibler, Macromolecules 13, 1602 (1980). 
${ }^{12}$ G. H. Fredrickson and E. Helfand, J. Chem. Phys. 87, 697 (1987).

${ }^{13}$ P. C. Hohenberg and B. I. Halperin, Rev. Mod. Phys. 49, 435 (1977).

${ }^{14}$ The main effect of the temperature quench is the change in the proximity to the spinodal temperature of the disordered state. The change in $T$ is usually small. Therefore, in most cases $T$ can be considered constant and we use $k_{B} T$ as the unit of energy.

${ }^{15}$ Y. Oono and Y. Shiwa, Mod. Phys. Lett. B 1, 49 (1987); M. Bahiana and Y. Oono, Phys. Rev. A 41, 6763 (1990).

${ }^{16}$ F. Liu and N. Goldenfeld, Phys. Rev. A 39, 4805 (1989).

${ }^{17}$ A. Chakrabarti, R. Toral, and J. D. Gunton, Phys. Rev. E 47, 3025 (1993).

${ }^{18}$ H. Chen and A. Chakrabarti, J. Chem. Phys. 108, 6897 (1998).

${ }^{19}$ S. Y. Qi and Z.-G. Wang, Phys. Rev. Lett. 76, 1679 (1996); S. Y. Qi and Z.-G. Wang, Phys. Rev. E 55, 1682 (1997).

${ }^{20}$ H. Zhang, J. Zhang, Y. Yang, and X. Zhou, J. Chem. Phys. 106, 784 (1997).

${ }^{21}$ A.-C. Shi, J. Noolandi, and R. C. Desai, Macromolecules 29, 6487 (1996); A.-C. Shi and J. Noolandi, Polymer 39, 4649 (1998).

${ }^{22}$ T. Ohta and K. Kawasaki, Macromolecules 19, 2621 (1986).

${ }^{23}$ If this form of $\Gamma_{2}$ is obtained by interpolating between the small and large $k$ asymptotics, then $b=(\sqrt{3} / 2)\left[f_{A}\left(1-f_{A}\right)\right]^{-3 / 2}$.

${ }^{24}$ J. W. Cahn and J. E. Hilliard, J. Chem. Phys. 28, 258 (1958).

${ }^{25}$ P.-G. de Gennes and J. Prost, The Physics of Liquid Crystals, 2nd ed.
(Clarendon, Oxford, 1993).

${ }^{26}$ P. M. Chaikin and T. C. Lubensky, Principles of Condensed Matter Physics (Cambridge University Press, Cambridge, 1995).

${ }^{27}$ Z.-G. Wang, J. Chem. Phys. 100, 2298 (1994).

${ }^{28}$ J. L. Goveas and S. T. Milner, Macromolecules 30, 2605 (1997).

${ }^{29}$ G. H. Fredrickson and K. Binder, J. Chem. Phys. 91, 7265 (1989).

${ }^{30}$ T. Hashimoto and N. Sakamoto, Macromolecules 28, 4779 (1995); N. Sakamoto and T. Hashimoto, ibid. 31, 3815 (1998).

${ }^{31}$ M. C. Newstein, B. A. Garetz, H. J. Dai, and N. P. Balsara, Macromolecules 28, 4587 (1995); H. J. Dai, N. P. Balsara, B. A. Garetz, and M. C. Newstein, Phys. Rev. Lett. 77, 3677 (1996); M. C. Newstein, B. A. Garetz, N. P. Balsara, M. Y. Chang, and H. J. Dai, Macromolecules 31, 64 (1998).

32 G. Floudas, S. Pispas, N. Hadjchristidis, T. Pakula, and I. Erukhimovich, Macromolecules 29, 4142 (1996).

${ }^{33}$ D. A. Hajduk, T. Tepe, H. Takenouchi, M. Tirrell, S. F. Bates, K. Almdal, and K. Mortensen, J. Chem. Phys. 108, 326 (1998).

${ }^{34}$ N. P. Balsara, B. A. Garetz, M. C. Newstein, B. J. Bauer, and T. J. Prosa, Macromolecules 31, 7668 (1998)

${ }^{35}$ M. A. Modi, R. Krishnamoorti, M. F. Tse, and H.-C. Wang, Macromolecules 32, 4088 (1999). 